\title{
How NFTs Influence Society: A Look at Scarcity Mindset, Generational Gaps in Education, and the Impact on the Environment
}

\author{
Ariana Schrader-Rank
}

\begin{abstract}
I propose that non fungible tokens (NFTs) will affect the greater public, and specifically the art market, at an exponential rate due to three factors. The first, scarcity mindset, drives the human sense of urgency for a particular commodity (Garvey, 2021). The next factor is the potential use of NFTs in real world applications or throughout the economy. The attraction of NFTs is that they are indiscriminate and allow anyone from various socioeconomic backgrounds to buy in. As scarcity seemingly increases, NFTs appear to be a good investment; but are there real world applications or do they merely exist within the virtual realm? The last determinant I would like to explore is the environmental impact of NFTs on the physical world. When comparing virtual 'tokens' used to fund digital art to paper money exchanged for a piece of physical artwork in concrete space, theoretically the former is much less detrimental to society. However, through practice-led research, I have conducted a six month investigation from May 2021 through October 2021 to uncover the true ramifications NFTs have on the world.

The cumulative evidence I have gathered in this paper is intended to help others understand art at a fundamental level. It's imperative to educate myself and others about the implications of NFTs on the arts, particularly because they are still in their burgeoning growth. As a future educator, I feel that understanding how young people interact with new and upcoming art trends is an invaluable resource to apply to a classroom setting.
\end{abstract}

\section{Overview}

First introduced in 2014, but not widely available until 2017, non fungible tokens (NFTs) are unique codes that represent strings of metadata. If an item is fungible it can be interchanged easily, not unlike paper money. In addition, if something is non fungible it is unique and cannot be copied. Mirriam-Webster added Non-Fungible Token to their dictionary in 2021, with the definition, "a unique digital identifier that cannot be copied, substituted, or subdivided, that is 
recorded in a blockchain, and that is used to certify authenticity and ownership (as a specific digital asset and specific rights relating to it)" (Mirriam-Webster, 2021). A blockchain is a database with distinguishing characteristics, indicated by the way it stores information. As new data (such as an NFT) enters, it is stored in a fresh block, and once that block is full of information it is automatically chained to the previous block. This allows information entering the blockchain to couple together in sequential order, and create a digital, immutable ledger. Blockchain technology is currently decentralized and self-sufficient with no single user having complete power, which is why it has grown in popularity over the last few years (Lafoucade, Lombard-Platet, 2020). Along with NFTs, encryption-based currencies (cryptocurrencies) have gained popularity on the blockchain as a result of the E-commerce era. Cryptocurrencies are decentralized and have indeterminate value, based on whether or not the public thinks they have worth (not dissimilar to equity options). Cryptocurrencies subsequently purchase NFTs and a large majority of them are found on the Ethereum blockchain.

Until recently, cryptocurrencies were 'mined;' the process through which Bitcoin and other Proof-of-Work systems entered new currency into circulation. Through Proof-of-Work (PoW) mining, the network server shares a complex puzzle with miners, or those users who are trying to gain access to more cryptocurrency. The miners send different solutions of the puzzle to the network server, and a reward of digital monetary value is awarded to the person (miner) who solves the algorithm first. PoW uses quite a large amount of energy from central processing units (CPU) when users are mining. Miners sometimes need to increase their CPU effort in order to attempt to solve the algorithm several times. This is detrimental to energy consumption and increases their contribution to the carbon footprint ( Bitcoin Network Power Demand, 2021). Proof-of-Stake (PoS) however, is a peer-to-peer currency ownership, and operates under a 
fundamentally different principle. PoS requires validators, and you can 'validate' based on how many coins you have in your possession. With PoS, as one raises their stakes, their probability of being chosen as the next validator increases. PoS uses less computing power than PoW, and as cryptocurrency and NFT usage rises, we must begin looking at the environmental impact of blockchain (AlAhmad, Al-Saleh, AlMasoud, 2018, p.1255).

Along with environmental consequences, some people have noticed that non fungible tokens have been gaining popularity with increasinging magnitude, and shortage of supply mindset. Not to be confused with actual scarcity of resources, this mindset is driving the general population to overbuy, overspend, and create a vast market throughout the blockchain for NFTs with cryptocurrency as the payment. This hyperinflation has caused society as a whole to perceive a scarcity mindset and allow the NFT market to expand exponentially. Rob Massey, CPA, Partner, and Global and U.S. Tax Blockchain and Digital Assets Leader at Deloitte Tax LLP, adds that "value is based on perceptions of owners and buyers, scarcity, access, and the distribution channel. There are no physical limitations on NFTs..." (Murphy, 2021). As a result of this market boom, potential investors in the NFT market are anyone who has the funds to buy in. While equity of access is what the Internet has always striven for, there is a massive gap in technical knowledge. Due to the networking PoS and PoW require in order to gain more cryptocurrency, users should be better informed about what they are purchasing and eventually acquiring. After spending exorbitant amounts of money on digital assets, some patrons still don't fully understand that they are validating, mining, or even going through the process to contribute to the larger ecological footprint. In terms of educational value; technology is changing and advancing rapidly, leading me to believe that interdisciplinary digital education through middle and high school courses should be required. 


\section{Introduction}

We live in a capitalist society, where supply drives demand. If you cut off the supply or create more demand, the society will be forced to acknowledge that there is a shortage and scarcity-whether real or perceived- occurs. On February 16, 2021 the artist Beeple (also known as Mike Winkelmann), sold his Everydays: The First 5000 Days through Christie's auction house for a record breaking $\$ 69.3$ million in cryptocurrency (Romeo, 2021). No auction house had previously allowed digital files built on blockchain technology to be sold, nor had they ever accepted cryptocurrency to be used as a payment method. During the Covid-19 pandemic of 2020 , Bitcoin shares had already increased to nearly $\$ 24,000$, which was a $224 \%$ rise. The days following this sale were a whirlwind for media outlets. Only two months after Beeple's momentous art sale, on April 14, 2021 Bitcoin reached a new peak of more than $\$ 64,000$ per share (Bitcoin Price, 2021). As a result, NFTs, new cryptocurrencies, and the blockchain were soon on everyone's radar.

During the global shutdown due to the Covid-19 pandemic, many struggling artists turned to digital media as a means to continue producing art and sustain their livelihoods. Not only visual artists, but musicians, writers, videographers, and other creative professions got wind of NFTs and what a benefit they could be to the art community. While the world was seemingly shut down to in-person activities, the digital universe was surviving, thriving and entering new paradigms. The downside that users and artists encountered was that the initial process of programming an NFT was bulky and complicated. Unless the artist had coding knowledge or 
possessed specific graphic design skills, the channels to generate a single NFT were quite frustrating. Many artists seemed to feel this way, and as a result, software engineer and multi-platform founder, Tim Kang, came onto the scene.

Using the Internet as a revenue source during a global pandemic was a clever, new and modern way for artists to continue their livelihoods. However, it did come into question whether or not the benefits outweighed the drawbacks. Kang created the Sevens Foundation and launched it "as a nonprofit organization dedicated to elevating artists" (Sevens Foundation, 2021). What Tim Kang and his team of donors have done is to empower artists by helping them to turn their artwork digital and then "mint" the work for free or low cost. Minting is the technical term for making work appear on the blockchain. Although like anything on the blockchain, minting is usually associated with being timely, cumbersome and costing real money. If you were to mint based completely on the blockchain, you would need to acquire a digital wallet, either through download or an app. Then you would need to establish a crypto-exchange account for said-wallet and put money into the wallet. Afterward, you would actually generate the NFT, which could be any music, video, or image file that you want, and upload it to your pre-funded digital wallet; however, in order to post your NFT to a marketplace you must pay the minting fee. The minting fee is generally proportional, and increases as the price of your NFT increases, and additionally it may depend on which marketplace you use (Genc, 2021). This is why the Sevens Foundation was revolutionary for artists and makers alike. Suddenly artists were put on an even playing field and could apply for a Sevens Grant to receive scholarships for minting fees, and help with publishing new digital works (Vartanian, 2021).

NFTs exist solely in the digital realm, and rely either on the PoW or PoS model to enter the blockchain. As aforementioned, PoW requires extensive mining power and resources, while 
PoS aims to address the same issues and is scalable while maintaining cost, rather than increasing it (Alahmad, AlMasoud, Al-Saleh, 2018). The world was in shock when news outlets initially publicized how much energy Bitcoin was actually consuming (Criddle, 2021). According to the analysis that came through the Cambridge Bitcoin Electricity Consumption Index (CBECI), acknowledged as of July, 2021, Bitcoin's electricity consumption was 121.05 terawatt-hours (TWh). This single figure surpassed the entire country of Argentina at $121 \mathrm{TWh}$. The CBECI estimates that Bitcoin uses an average daily network power demand of 13.39 Gigawatts (GW). To put that in perspective, just $1 \mathrm{GW}$ is equal to 3.125 million photovoltaic panels, 364 utility-scale wind turbines, 110 million LED light bulbs, or 2,000 Corvette Z06s's (How Much Power is 1 Gigawatt, 2019). Artists and investors alike quickly floated the question of how they would keep up with increasing demand for NFTs, cryptocurrency, and the constant need for power throughout the blockchain, without permanently damaging the Earth.

One answer may lie within Greenidge Generation Holdings, a relatively young, privately owned company, with plants located in Dresden, NY and Spartanburg, SC, and a projected plant in Texas. Greenidge Generation Holdings purchased old power plants and converted some to burn natural gas, and some nuclear power, with the hopes of mining their own Bitcoin at a significant discount (Martin, Nauman, 2021). On their own public website, Greenidge Holdings has boasted that "two thirds of [our] electricity is sourced from zero carbon sources such as nuclear power" (Environmental Stewardship, 2021). While Greenidge has been helping PoW sources like Bitcoin become carbon neutral, its new sources of power are equally as concerning as traditional sources. Greenidge uses nuclear power and natural gas, which makes it difficult for environmentalists to support the switch to this mode of mining Bitcoin, even if the company has proven they are carbon neutral. The global community has increasing awareness of the power 
usage of PoW and PoS, but there has been considerably less talk about how our societies will continue to contemporize in order to accomodate the technology of NFTs and cryptocurrency.

\section{Body}

On June 8, 2021, the President of El Salvador, Nayib Bukele, created a new Bitcoin Law, sent it to The Legislative Assembly of the Republic of El Salvador, and then proceeded to tweet the document in both English and Spanish. In this Bitcoin Law, President Nayib Bukele asserted that "IV. approximately seventy percent of the population [of El Salvador] does not have access to traditional financial services. V. That in order to promote the economic growth of the nation, it is necessary to authorize the circulation of a digital currency whose value answers exclusively to free-market criteria...” Then on June 9, 2021, President Bukele, announced that his country would begin to use geothermal energy generated from volcanoes in order to mine Bitcoin, the country's new legal tender, alongside the U.S. dollar (Hernandez, 2021). While many see this as a victory for El Salvador, because this once disadvantaged country now has access to an open global monetary network, others only see problems. Bitcoin, along with nearly every other cryptocurrency, has a nebulous value, and it seems preposterous for an entire country to base itself on a currency that is unregulated. However, regulation is on the horizon. Several times over the past year, U.S. lawmakers have come together to propose legislation for the American cryptocurrency market (Haar, 2021). These attempts have been met with little success, but there is a new cryptocurrency tax amendment bill that has been put forward, known as Publication 1544. Publication 1544 would require reporting directly to the U.S. Internal Revenue Services (IRS) if you receive more than $\$ 10,000$ from a crypto trade or transaction. If enacted, this bill would require the details of the sender to be reported to the IRS as well, which is very difficult to 
trace on the blockchain (Publication 1544, 2021). The IRS is trying to enact Publication 1544 because they see trading of cryptocurrency and buying and selling of NFTs as unearned profits of which the government should be receiving their fair portion. Anything owned for personal investment is considered a capital asset, including NFTs and cryptocurrency. When you sell your capital assets, the sale is a capital gain or capital loss, and a "capital gain rate of $15 \%$ applies if your taxable income is $\$ 80,000$ or more but less than $\$ 441,450$ for single filing. Some or all net capital gain may be taxed at $0 \%$ if your taxable income is less than $\$ 80,000$ " (Topic No. 409 Capital Gains and Losses, 2021). This means that some investors have found a new loophole around the U.S. capital gains tax, which is why the U.S. government has been so aggressive in seeking to pass Publication 1544 (Sigalos, 2021).

Buying and selling NFTs and cryptocurrency, strictly speaking, should be available to anyone with the means, but it has been largely benefiting the affluent in more recent months. As the NFT market grows and becomes more mainstream, artists have been able to market themselves to a wider audience, even if they have little background in digital arts. Well known public figures are also benefiting, which means business opportunities for smaller artists are potentially being taken away (Book, 2021). Major brand name recognition and high profile influencers promote transactions throughout big business. Now celebrities are not only creating limited NFTs but also branding their own "social currency." Social currency refers to specific cryptocurrency that a brand, community, or influencer releases in order to make their fans feel significant and part of their niche fandom. One example is Terry Crews' social currency \$POWER. Fans of Terry Crews that engage with him online platforms such as Discord, learn of his currency because he offers special access channels if they buy into certain levels of \$POWER. Crews has even stated in Discord streams, "If I give you \$POWER, you own a piece 
of me" (Rose Dickey, 2021). NFTs have grabbed the attention of the ultra wealthy, so along with Terry Crews, media personality Paris Hilton, American entrepreneur Mark Cuban, Canadian musician Grimes, and entrepreneur and CEO of SpaceX Elon Musk are all names that appear alongside NFT and cryptocurrency news almost every day. When Grimes sold her NFT Earth collection back in February 2021, she netted a sum total of $\$ 5.8$ million in a matter of minutes. Her fans expected resale values to exponentially rise. However, as of November 16, 2021 the NFTs issued by Grimes have decreased in value by $84 \%$ (Kharif, Bhasin, 2021). When I interviewed Technology Innovation Consultant, Peg Samuel, she mentioned the Clubhouse App (Peg Samuel, 2021). In our conversation Samuel brought up American DJ and EDM producer 3LAU (pronounced blau) who has been streaming music through Clubhouse. At the time of our interview, Clubhouse was an invite-only app where users can host private discussion or stream concerts. Due to the exclusivity of the app, only those who were invited could be a part of the conversation that was occurring early on, about NFTs and cryptocurrency. Blau's music is so popular, when his first ever NFT-based albums were released they sold in less than a day for $\$ 11.6$ million. This brings up the idea of scarcity once again, because even as the blockchain becomes more accepted, and NFTs go down in value, only the elite were being welcomed into Clubhouse for a 3LAU concert. This exclusivity allows artists to gain an intense following and raise prices, in order to create sensational experiences that their fans would pay huge amounts for (Samuel, P., June 15, 2021). Or at least they would have paid well previously. On July 21, 2021, co-founders of Clubhouse Paul Davison and Rohan Seth announced that the app would no longer be invite-only. With a waiting list of more than ten million people (including myself), Clubhouse realized that in order to compete with larger social spheres such as Twitter, they needed to open up their access to the greater whole (Carman, 2021). 
As a result, of the unforeseen influx of newcomers to the NFT and cyrotcurrecy scene, the market was once again on fire. Bored Ape Yacht Club (BAYC) officially launched on April 30, 2021, which offered 10,000 unique avatar versions of humanoid-looking ape NFTs. One day after the launch, all 10,000 NFTs had been sold on OpenSea, the world's largest NFT marketplace (Chayka, 2021). Due to the success of the CryptoPunks which were launched by LarvaLabs, both buyers and sellers have been especially successful vending multiple versions of NFTs. A twelve year old coder, Benyamin Ahmed, who created 3,350 NFTs, naming them his Weird Whale series, sold them out completely despite buyers being unable to see which version they were bidding on until they won their auction (Locke, 2021). The idea of the digital multiple is intriguing, and new versions are popping up every day (See Figure 1). The multiple itself is not new, and has existed for centuries through printmaking. Now with NFTs the artist can digitally track the authenticity of sales, apply fractional ownership, and resell within moments. Editions of multiple NFTs are sought-after, which puts digital artists at a disadvantage, and coders in the forefront. Coders can easily create computer generated images, and tweak code including how many iterations they want. This is a disservice to digital artists who create their unique artwork, and want to show off their piece's individuality. 


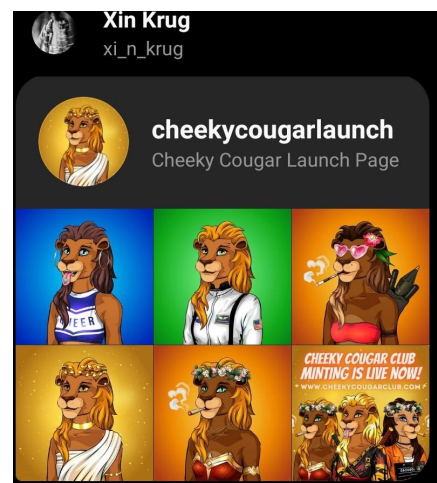

As I said..

These guys sold out their prior project (Cheeky Lion Club) for $\$ 2.5 \mathrm{~m}$, and this one won't be

any different, specially with the enormous community behind

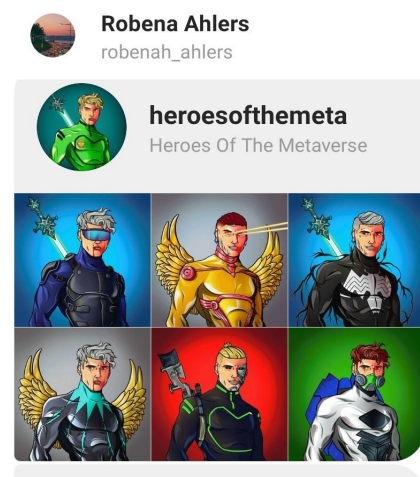

Is it you?

$\mathrm{Hi}$ !

I joined this new project a while ago, it's really amazing!

This NFT is the future, you can earn $5000 \$ /$ month just by

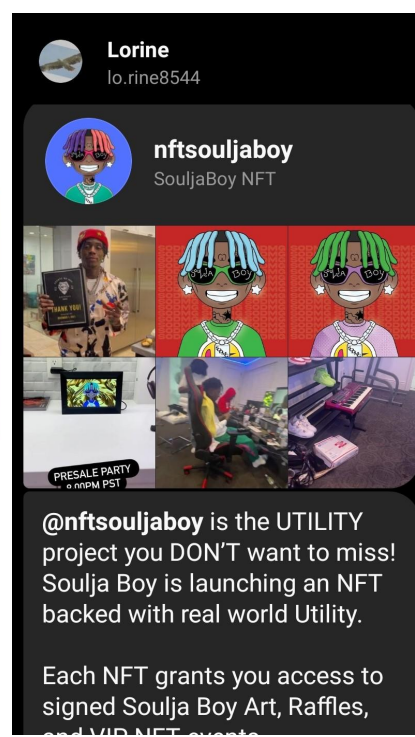

signed Soulja Boy Art, Raffles,

Figure 1.

From creation to marketing, all the way to profit, it seems as if selling art either digitally or in real life takes time, effort and money. Tangible art sales have many advantages. One major disadvantage is that the artist can only profit the first time their art is sold. The difference with NFTs and online galleries or auction houses is that creators are able to set royalties on their artwork. The sharing economy promotes collaborative consumption of under-utilized goods; and there is an assumption that many artists and galleries will be pushed to go completely digital with their work. When I spoke to Peg Samuel she expressed her interest in fractional ownership of artworks on the blockchain. Fractional ownership is sharing the right of possession of a luxury good, which in the case of many NFTs. Fractional ownership means that artists can benefit from the sales of their artwork several times. Artists and creators alike have been utilizing fractional ownership through buying into masterworks, including the likes of Jean-Michel Basquiat, Andy Warhol, Banksy and others (Pasimeni, 2020). Teaching students in the art classroom about fractional ownership, as well as how to promote themselves as artists, is an invaluable, necessary tool. Many artists continue to struggle to gain recognition, but through the metaverse, their 
artwork might have a higher profile. Artwork has the ability to sell if it is well-liked on a well-known blockchain platform. However, one issue that arises is that blockchain platforms are not connected, which is what most people refer to as interoperability. As a result, Polkadot was created as a way to solve some of the main software exchange issues between platforms (Polkadot, 2021).

The Polkadot Network allows for cross-blockchain transactions and transfers. Polkadot is a platform built on parachains. Parachains are independent platforms that connect any type of data through a substrate blockchain framework. Both Polkadot and another similar network, Kusama, are decentralized (Kusama, 2021). Decentralization means the processes of interactions are fully controlled by the users or developers within the network, who work together to propose changes to the network (Polkadot, 2021). Kusama and Polkadot are considered cousins and were built upon similar code structures. In fact, according to the Polkadot Network (2021),

"Kusama and Polkadot are independent, standalone networks built on very similar codebases, but Kusama has faster governance parameters and lower barriers to entry. While Kusama is wild and fast, Polkadot is more conservative, prioritizing stability and dependability, with slower, more methodical governance and upgrade processes. Kusama is great for bold experimentation and early-stage deployment. Polkadot is designed for stable execution of risk-averse, high-value applications."

Both the Polkadot Network and Kusama are uniting our global network unlike ever before. Despite the Internet existing for forty years now, there is a contemporary concept of E-learning as a tool for students and adults alike. This is a reaction to the overwhelming majority of those who now own personal computers, cellphones, laptops, and the ease of access to the Internet both in school and out. The "semantic web" also known as Web 3.0, is the culmination of the Internet environment up until today. Under Web 3.0, data isn't owned but shared, there is greater 
connectivity and interoperability (such as Kusama and Polkadot), and artificial intelligence to control the blockchains or nodes (Acikgul Firat, Firat, 2021, p. 149). In my interview with Architectural Designer and Founder of Sentient Artworks, Joshua Bacigalupi, the discussion of Web 3.0 was further expanded upon. Bacigalupi specifically mentioned the Internet of Things (IoT) which is the concept of societies continually incorporating newer technologies, smart industries, pollution control and being fully interconnected via the Internet (Web 3.0) (Kuwar, Tiwari, Zymbler, 2019). This is significant in terms of teaching in the art classroom, because Bacigalupi determined that in an ideal society, the framework would be a triple bottom line economy. In the triple bottom line, the structure is ideally equal parts socially, economically and environmentally developed (Hammer, Pivo, 2016). Bacigalupi tweaked the diagram to show the civic network, or community based groups that one interacts with, the physical network which would encompass physical artworks, and the digital network which would include the blockchain and, among other things; IoT. He went on to mention how this framework is only effective when NFTs are part of the larger solution, and when the entire society is accepting. One point Bacigalupi identified that was distinct in my mind was the concept of NFTs as a basis of daily interactions (Joshua Bacigalupi, 2021).

Challenging the proposal that all members in a community would always work toward the benefit of their community or group was Elinor Ostrom, who in 1990 supported these beliefs through her book, Governing the Commons (pp.6-8). Ostrom argued that often people act in self interest, and in almost every community there are individuals who are productive while others are freeloaders. Joshua Bacigalupi built upon Ostrom's theory saying that societies need local emerging governance. He let me know that as technology advances, "members in a community will work together to create artwork... to be able to use NFTs as a way of tracking art and media 
but also in a traditional workforce environment" (Joshua Bacigalupi, 2021). This presents the idea that resumes would be borderline automated, as digital infrastructure would accompany and update it daily based on your human interactions and life skills. Incorporating this technology into our future societies, even if in a smaller capacity, would be a contribution to start transforming communities.

Based on my interactions with Samuel and Bacigalupi, I was beginning to recognize that our society, with NFTs on the rise and the IoT at the forefront of technology, should start addressing our economic policy with more of an institutionalist economic view. Institutional economists are interested in the links between technology, culture and money (Hodgson, 2001). Of course, NFTs as part of a community's digital infrastructure would not be possible if 1 : the society did not accept it and 2: the individuals within the society were not educated thoroughly. The second point was brought to light when I interviewed Partner \& Chief Climate Officer of Convincing Crypto, Gianna Lum. On Lum's YouTube page, also named Convincing Crypto, she mentioned the work that Cardano, an environmentally sustainable blockchain platform, is doing (Lum, 2021). Specifically, Cardano Input Output Hong Hong (IOHK), has partnered with the European Business Union (EBU) and has been offering programs that make digital education more affordable and available to 2000+ students in 25 countries on the African continent (Ahern, 2021). We will need to continue to educate students about rapidly changing technology and digital infrastructure, not just in Africa but worldwide. In pursuance of a technologically globally educated society, cryptocurrency, the blockchain and NFTs must all be acknowledged and taught in school.

During 2021, the U.S. Congress introduced 18 separate bills addressing cryptocurrency, the blockchain and NFT sales. The introduction of these bills was for a few different reasons, but 
largely the lack of control Congress has felt over the digital world. There is also currently no legal way to regulate crypto throughout the blockchain. Some members of Congress feel that with technology advancing so rapidly, the government must do their part to keep up, and intervene through education and law (Brett, 2021). During the six months I researched NFTs and cryptocurrency, several bills and amendments were introduced, including one crypto amendment put forth by Senators Pat Toomey (R-Pa), Cynthia Lummis (R-Wy) and Senate Finance Committee Chair Ron Wyden (D-Or) which was unsupported by the Biden administration as recently as September, 2021. However, on November 15, 2021 President Joe Biden signed the infrastructure bill into law which contained the cryptocurrency exchange reporting requirement. This new law requires any cryptocurrency exchange over $\$ 10,000$ to be reported with a 1099-B (Peterbaugh, 2021). However, there are complaints about the vague wording of the bill. If not self-reported correctly to the IRS, any person receiving digital assets of $\$ 10,000$ (NFTs, cryptocurrency, etc.) can be charged with a felony (Sutherland, 2021). It is important to teach individuals about the basics of cryptocurrency and investing in NFTs before they commit to participation or purchase. Someone who gains notoriety as an artist overnight through an online platform might not be aware that they have to report their crypto at certain income brackets. It is at this time I am again advocating for curriculum changes to be made in all secondary education that provides basic insight into technology, the arts and cryptocurrency.

\section{Conclusion}

I proposed that NFTs would affect the greater public, specifically the art market, at an exponential rate due to three factors. The first being scarcity mindset which drives human sense of urgency for a particular commodity. The next factor being how NFTs might be used in real 
world applications or throughout the economy. The last determinant I explored was the environmental impact of NFTs on the physical world. Through practice-led research over the course of six months between May 2021- October 2021, I dug deeply into the true ramifications that NFTs have had on the world thus far. The cumulative evidence I found out about NFTs, cryptocurrency and the blockchain affects how art is viewed as an object or commerce and consumption. Educating myself and others about the implications and expansion of NFTs on the arts is imperative, particularly now as they are still new to so many in their burgeoning period of growth. Due to the majority of the general public being unfamiliar with the blockchain, cryptocurrencies, and having just been introduced to NFTs, there is currently a massive gap within generational instruction. As a future art educator, understanding how students view and interact with new and upcoming art trends is an invaluable resource to apply to the classroom setting. I looked into the effects of the NFT marketplace and how influential it is to future generations who view and value art, and found a great deal of interest from all over. I have found evidence that technology in conjunction with art (i.e. NFTs) is evolving at a rapid pace. Society is taking notice and we need to continue to educate ourselves on both the benefits and detriments in order to fully inform ourselves of what is to come. 


\section{References}

Acikgul Firat, E., Firat, S. (2021). Web 3.0 in Learning Environments: A Systematic Review. Turkish Online Journal of Distance Education. 22(1). pp. 148-163.

Ahern, N. (2021). Making Education in Africa More Accessible, Affordable, and Equitable. IOHK.io. Retrieved from https://iohk.io/en/blog/posts/2021/08/24/making-education-in-africa-more-accessible-affo rdable-and-equitable/

AlAhmad, M.A., Al-Saleh, A., AlMasoud, F.A. (2018). Comparison Between PoW and PoS Systems of Cryptocurrency. Indonesian Journal of Electrical Engineering and Computer Science. 10 (3). Pp. 1251-1256.

Bacigalupi, J. (June 22, 2021). Personal Interview.

Bitcoin Network Power Demand. Cambridge Bitcoin Electricity Consumption Index. Retrieved from https://cbeci.org/index on September 20, 2021.

Book, A. (2021). NFTs Are Just Another Way for the Rich to Get Richer. The Pourquoi Pas. Retrieved from https://www.thepourquoipas.com/post/nfts-are-just-another-way-for-the-rich-to-get-richer

Brett, J. (2021). Congress Has Introduced 18 Bills On Crypto and Blockchain in 2021. Forbes.com. Retrieved from https://www.forbes.com/sites/jasonbrett/2021/08/22/congress-has-introduced-18-new-bill s-on-crypto-and-blockchain-in-2021/?sh=337db61263b0

Carman, A. (2021). Clubhouse is No Longer Invite-Only. The Verge. Retrieved from https://www.theverge.com/2021/7/21/22586845/clubhouse-open-beta-invite-access-andro Id-ios

Chayka, K. (2021). Why Bored Ape Avatars Are Taking Over Twitter. New Yorker. Retrieved from https://www.newyorker.com/culture/infinite-scroll/why-bored-ape-avatars-are-taking-ove r-twitter

Coinbase.com. (2021). Bitcoin Price. Retrieved from https://www.coinbase.com/price/bitcoin on November 15, 2021. 
Criddle, C. (2021). Bitcoin Consumes 'More Electricity Than Argentina.' BBC News. Retrieved from https://www.bbc.com/news/technology-56012952

Environmental Stewardship. Greenidge Generation Holdings. Retrieved from https://greenidge.com/ on October 10, 2021.

Garvey, C. (2021). How Scarcity Mindset Impacts Artists. Retrieved from http://www.christine-garvey.com/news/2019/9/19/how-scarcity-mindset-impacts-artists?r $\mathrm{q}=$ scarcity

Genc, E. (2021). Beginner's Guide to NFTs: How to Mint a Non-Fungible Token on Ethereum. Decrypt.com. Retrieved from

Haar, R. (2021). U.S. Officials Send Mixed Messages on Crypto Regulation. Here's What it All Means for Investors. Time. Retrieved from https://time.com/nextadvisor/investing/cryptocurrency/crypto-regulation-talks-heat-up/

Hammer, J., Pivo, G. (2016). The Triple Bottom Line and Sustainable Economic Development Theory and Practice. Sage Journals. 31 (1).

Hernandez, J. (2021). El Salvador Plans to Use Electricity Generated from Volcanoes to Mine Bitcoin. NPR. Retrieved from https://www.npr.org/2021/06/11/1005231250/el-salvador-plans-to-use-electricity-generat Ed-from-volcanoes-to-mine-bitcoin

Hodgson, G.M., (2001). Institutional Economic Thought. International Encyclopedia of the Social \& Behavioral Sciences. Berlin: Max Planck Institute for Human Development.

Internal Revenue Service. (2021). Topic No. 409 Capital Gains and Losses. Retrieved from https://www.irs.gov/taxtopics/tc409 on July 22, 2021.

Kusama. (2021). Parachains Are Here. Retrieved from https://kusama.network/ on October 12, 2021

Locke, T. (2021). This 12-Year-Old Coder Is Set to Earn Over \$400,000 After About 2 Months Selling NFTs. CNBC News. Retrieved from https://www.cnbc.com/2021/08/25/12-year-old-coder-made-6-figures-selling-weird-whal es-nfts.html 
Lum, G. (September, 17, 2021). Personal Interview.

Martin, K. and Nauman, B. (2021). Bitcoins Growing Energy Problem: It's a Dirty Currency. Financial Times. Retrieved from https://www.ft.com/content/1aecb2db-8f61-427c-a413-3b929291c8ac

Mirriam-Webster. Non-Fungible Token. Retrieved from https://www.merriam-webster.com/dictionary/NFT on November 11, 2021.

Murphy, M.L. (2021). NFTs Come With Big Valuation Challenges. Journal of Accountancy.

Office of Energy Efficiency \& Renewable Energy. (2021). How Much Power is 1 Gigawatt? Retrieved from https://www.energy.gov/eere/articles/how-much-power-1-gigawatt on September 20, 2021.

Ostrom, E. (1990). Governing the Commons: The Evolution of Institutions for Collective Action. Cambridge: Cambridge University Press. pp.6-8.

Khari, O. and Bhasin, K. (2021). An NFT Issued by the Musician Grimes Plunges in Value by $84 \%$ as Celebrity-Minted Tokens Struggle in the Market. Fortune.com Retrieved from https://fortune.com/2021/11/16/nft-celebrity-value-plunge-investment-grimes-asap-rocky -john-cena/

Lafourcade, P., Lombard-Platet, M. (2020). About Blockchain Interoperability. Elsevier. 161.

Pasimeni, F. (2020). The Origin of the Sharing Economy Meets the Legacy of Fractional Ownership. ResearchGate. DOI:10.2139/ssrn. 3768456

Peterbaugh, J. (2021). Two Things Crypto Investors Should Know About the Infrastructure Bill President Biden Signed. Time.com. Retrieved from https:/time.com/nextadvisor/investing/cryptocurrency/infrastructure-bill-crypto-taxes/

Polkadot. (2021). Retrieved from https://polkadot.network/ on September 21, 2021.

Publication 1544. Department of the Treasury Internal Revenue Service. Retrieved from https://www.irs.gov/pub/irs-pdf/p1544.pdf on July 22, 2021.

Romeo, J. (2021). What's the Deal With Crypto Art? JSTOR Daily. Retrieved from https://daily.jstor.org/whats-the-deal-with-crypto-art/ 
Rose Dickey, M. (2021). Why Terry Crews is Launching a Social Currency. TechCrunch. Retrieved from https://techcrunch.com/2021/03/10/why-terry-crews-is-launching-a-social-currency/

Samuel, P. (June 15, 2021). Personal Interview.

Sevens Foundation. (2021). Grants Program. Retrieved from https://grants.art/

Sigalos, M. (2021). A Tax Loophole is Helping Bitcoin Holders Save Tons of Cash by Avoiding Federal Taxes. CNBC. Retrieved from https://www.cnbc.com/2021/07/25/tax-loophole-wash-sale-rules-dont-apply-to-bitcoin-et Hereum-dogecoin.html

Sutherland, A. (2021). Research Report on Tax Code 60501 and Digital Assets. Proof of Stake Alliance. Retrieved from https://www.proofofstakealliance.org/wp-content/uploads/2021/09/Research-Report-on-T ax-Code-6050I-and-Digital-Assets.pdf

Vartanian, H. (2021). Collector Tim Kang Talks About His Love of NFTs. Hyperallergic.com. Retrieved from https://hyperallergic.com/649781/collector-tim-kang-talks-about-his-love-of-nfts/

Kuwar, S., Tiwari, P., Zymbler, M. (2019). Internet of Things is a Revolutionary Approach for Future Technology Enhancement: A Review. Journal of Big Data. 6 (111). 